International Journal of Pure and Applied Mathematics

Volume 93 No. 4 2014, 525-540

ISSN: 1311-8080 (printed version); ISSN: 1314-3395 (on-line version)

url: http://www.ijpam.eu

doi: http://dx.doi.org/10.12732/ijpam.v93i4.4

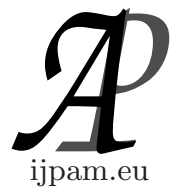

\title{
SOME EFFICIENT IMPLEMENTATION SCHEMES FOR IMPLICIT RUNGE-KUTTA METHODS
}

\author{
R. Vigneswaran ${ }^{1}$, S. Kajanthan ${ }^{2}$ \\ ${ }^{1,2}$ Department of Mathematics and Statistics \\ Faculty of Science \\ University of Jaffna \\ SRI LANKA
}

\begin{abstract}
Several iteration schemes have been proposed to solve the nonlinear equations arising in the implementation of implicit Runge-Kutta methods. As an alternative to the modified Newton scheme, some iteration schemes with reduced linear algebra costs have been proposed A scheme of this type proposed in [9] avoids expensive vector transformations and is computationally more efficient. The rate of convergence of this scheme is examined in [9] when it is applied to the scalar test differential equation $x^{\prime}=q x$ and the convergence rate depends on the spectral radius of the iteration matrix $M(z)$, a function of $z=h q$, where $h$ is the step-length. In this scheme, we require the spectral radius of $M(z)$ to be zero at $z=0$ and at $z=\infty$ in the $z$-plane in order to improve the rate of convergence of the scheme. New schemes with parameters are obtained for three-stage and four-stage Gauss methods. Numerical experiments are carried out to confirm the results obtained here.
\end{abstract}

AMS Subject Classification: 65L04, 65L05

Key Words: implementation, Gauss methods, rate of convergence, stiff systems

\section{Backround}

Let us consider an initial value problem for stiff system of $n(\geq 1)$ ordinary

Received: January 3, 2014

(c) 2014 Academic Publications, Ltd.

${ }^{\S}$ Correspondence author url: www.acadpubl.eu 
differential equations

$$
x^{\prime}=f(x(t)), \quad x\left(t_{0}\right)=x_{0}, \quad f: \mathbb{R}^{n} \rightarrow \mathbb{R}^{n},
$$

where $f$ is assumed to be as smooth as necessary. An $s$-stage implicit RungeKutta method computes an approximation $x_{r+1}$ to the solution $x\left(t_{r+1}\right)$ at grid point $t_{r+1}=t_{r}+h$ by

$$
x_{r+1}=x_{r}+h \sum_{i=1}^{s} b_{i} f\left(y_{i}\right)
$$

where the internal approximations $y_{1}, y_{2}, \cdots, y_{s}$ satisfy the $s n$ equations

$$
y_{i}=x_{r}+h \sum_{j=1}^{s} a_{i j} f\left(y_{j}\right), \quad i=1,2, \cdots, s
$$

$A=\left[a_{i j}\right]$ is the real coefficient matrix and $b=\left(b_{1}, b_{2}, \cdots, b_{s}\right)^{T}$ is the column vector of the Runge-Kutta method. Let $Y=y_{1} \oplus y_{2} \oplus \cdots \oplus y_{s} \in \mathbb{R}^{s n}$ and let $F(Y)=f\left(y_{1}\right) \oplus f\left(y_{2}\right) \oplus \cdots \oplus f\left(y_{s}\right) \in \mathbb{R}^{s n}$. Then equation (2) may be represented by the compact form

$$
Y=e \otimes x_{r}+h\left(A \otimes I_{n}\right) F(Y)
$$

where $e=(1,1, \cdots, 1)^{T}$ and $A \otimes I_{n}$ is the Kronecker product of the matrix $A$ with $n \times n$ identity matrix $I_{n}$ and, in general $A \otimes B=\left[a_{i j} B\right]$. This article deals with methods suitable for stiff systems so that the matrix $A$ is not strictly lower triangular and, in particular, is concerned with Gauss methods since they have highest order and good stability properties.

Equation (3) may be solved by a modified Newton iteration. Let $J$ be the Jacobian of $f$ evaluated at some recent point $x_{r}$, updated infrequently. The modified Newton scheme evaluates $Y^{1}, Y^{2}, Y^{3}, \cdots$, to satisfy

$$
\left(I_{s n}-h A \otimes J\right)\left(Y^{m}-Y^{m-1}\right)=D\left(Y^{m-1}\right), \quad m=1,2, \cdots,
$$

where $D$ is the approximation defect, $D(Z)=e \otimes x_{r}-Z+h\left(A \otimes I_{n}\right) F(Z)$. In each step of this iteration, a set of $s n$ linear equations has to be solved. Schemes have been developed, to solve equation (4), which use the fact that $J$ is constant [1], [6], [7]. In other schemes advantage is taken of the special forms of some implicit methods [2], [4], [5], [12].

In another approach, schemes based directly on iterative procedure have been developed [3], [8], [9], [10],[13],[21]. For a singly implicit method, there is a non-singular matrix $S$ so that $S^{-1} A S=\lambda\left(I_{s}-L\right)^{-1}$, where $L$ is zero except 
for some ones on the sub-diagonal. On applying this transformation, the scheme (4) becomes

$$
\begin{aligned}
{\left[I_{s} \otimes\left(I_{n}-h \lambda J\right)\right] E^{m} } & =\left[\left(I_{s}-L\right) S^{-1} \otimes I_{n}\right] D\left(Y^{m-1}\right)+\left(L \otimes I_{n}\right) E^{m} \\
Y^{m} & =Y^{m-1}+\left(S \otimes I_{n}\right) E^{m}, \quad m=1,2,3 \cdots
\end{aligned}
$$

Cooper and Butcher [8] proposed an iterative scheme, sacrificing superlinear convergence for reduced linear algebra cost, which may be regarded as a generalization of the scheme (5) for singly implicit methods. They considered the scheme

$$
\begin{aligned}
{\left[I_{s} \otimes\left(I_{n}-h \lambda J\right)\right] E^{m} } & =\left(B_{1} S^{-1} \otimes I_{n}\right) D\left(Y^{m-1}\right)+\left(L_{1} \otimes I_{n}\right) E^{m} \\
Y^{m} & =Y^{m-1}+\left(S \otimes I_{n}\right) E^{m}, \quad m=1,2, \cdots,
\end{aligned}
$$

where $B_{1}$ and $S$ are real $s \times s$ non-singular matrices and $L_{1}$ is strictly lower triangular matrix of order $s$, and $\lambda$ is a real constant. Cooper and Butcher [8] showed that successive over-relaxation may be applied to improve the rate of convergence for scalar test problem. Peat and Thomas [19], after extensive numerical experiments, concluded that the schemes proposed by Cooper and Butcher are, in general, the most efficient schemes for integration of stiff problems. Gladwell and Thomas [15] recommended this scheme for the two-stage Gauss method. Each step of the scheme (6) requires $s$ function evaluations and the solution of $s$ sets of $n$ linear equations. These $s$ sub-steps are performed in sequence and it is not possible to compute elements of $Y^{m}=y_{1}^{m} \oplus y_{2}^{m} \oplus \cdots \oplus y_{s}^{m}$ until all sub-steps are completed. Cooper and Vignesvaran [9] considered a scheme where these elements are obtained in sequence and the approximation defect is updated after each sub-step completed. Only one vector transformation is needed for each full step so that this scheme is more efficient. Another scheme was proposed by Cooper and Vignesvaran [10] in order to obtain improved rate of convergence, by adding extra sub-steps.Vigneswaran [20] obtained further improvement in the rate of convergence of the iteration scheme proposed in [10]. Gonzalez, Gonzalez and Montijano [16] proposed a scheme for Gauss methods using an iterative procedure of semi-implicit type in which the Jacobian does not appear explicitly. A scheme of this type was proposed in [17] in which convergence and stability properties of the scheme are discussed in detail. 


\section{Efficient Iteration Scheme}

Cooper and Vignesvaran [9] proposed the scheme

$$
\begin{aligned}
{\left[I_{s} \otimes\left(I_{n}-h \lambda J\right)\right] E^{m}=} & \left(L \otimes I_{n}\right)\left(e \otimes x_{r}-Y^{m}\right) \\
& +\left(U \otimes I_{n}\right)\left(e \otimes x_{r}-Y^{m-1}\right) \\
& +h\left(T \otimes I_{n}\right) F\left(Y^{m}\right) \\
& +h\left(R \otimes I_{n}\right) F\left(Y^{m-1}\right) \\
Y^{m}= & Y^{m-1}+E^{m}, m=1,2, \cdots,
\end{aligned}
$$

where $B$ is a real non-singular matrix such that $B=L+U$ and $B A=T+R$, $L$ and $T$ are strictly lower triangular matrices, $U$ and $R$ are upper triangular matrices, and $\lambda$ is a real constant. Cooper and Vignesvaran [9] showed that $D(Y)=0$ if the sequence $\left\{Y^{m}\right\}$ has a limit $Y$ and $f$ is continuous on $\mathbb{R}^{n}$. They observed that the scheme can be implemented efficiently by updating $Y^{m-1}$ and $F\left(Y^{m-1}\right)$ as soon as each element of $Y^{m}=y_{1}^{m} \oplus y_{2}^{m} \oplus \cdots \oplus y_{s}^{m}$ is computed. The work involved is no more than is needed to carry out an evaluation of $D\left(Y^{m-1}\right)$ followed by a transformation to $\left(B \otimes I_{n}\right) D\left(Y^{m-1}\right)$.

Cooper and Vignesvaran [9] tested the rate of convergence of this scheme when it is applied to the scalar test problem $x^{\prime}=q x$ with rapid convergence required for all $z \in \mathbb{C}^{-}$, where $\mathbb{C}^{-}=\{z \in \mathbb{C}:$ Re $\leq 0\}$. For this test problem, the scheme gives (7) gives

$$
Y-Y^{m}=M(z)\left(Y-Y^{m-1}\right), \quad m=1,2, \cdots,
$$

and the rate of convergence depends on the spectral radius $\rho[M(z)]$ of the iteration matrix

$$
M(z)=I_{s}-\left[\left(I_{s}+L-z\left(\lambda I_{s}+T\right)\right]^{-1} B\left(I_{s}-z A\right) .\right.
$$

Cooper and Vignesvaran[9] imposed the condition that the iteration matrix $M$ has only one non-zero eigenvalue $\phi$,

$$
\phi(z)=1-\beta \frac{\operatorname{det}\left(I_{s}-z A\right)}{(1-\lambda z)^{s}},
$$

so that the spectral raqdius, $\rho[M(z)]$, given by $\rho[M(z)]=|\phi(z)|$ and $\lambda$ and $\beta(=\operatorname{det} B)$ can be chosen to solve the problem

$$
\min _{\lambda, \beta} \max _{z \in \mathbb{C}^{-}} \rho[M(z)]
$$


To solve the minimization problem (10), when $\lambda>0$ it follows from (9) that $\phi$ is analytic and bounded on $\mathbb{C}^{-}$and hence $|\phi|$ attains its maximum on the imaginary axis $z=i y, y$ real. The polynomial $p$, defined by

$$
p(\omega)=|\phi(i y)|^{2}, \quad \omega=\frac{1}{1+(\lambda y)^{2}},
$$

is a polynimial of degree $s$. For a given method, the coefficients of $p$ depends on $\lambda$ and $\beta$ only and Cooper and Vignesvaran[9] obtained these parameters to minimize the maximum of $p$ on $[0,1]$ for the Gauss methods of order 4,6 and 8 respectively.

Consider the three-stage Gauss method with matrix of coefficients

$$
A=\left[\begin{array}{ccc}
\frac{5}{36} & \frac{2}{9}-\frac{\sqrt{15}}{15} & \frac{5}{36}-\frac{\sqrt{15}}{30} \\
\frac{5}{36}+\frac{\sqrt{15}}{24} & \frac{2}{9} & \frac{5}{36}-\frac{\sqrt{15}}{24} \\
\frac{5}{36}+\frac{\sqrt{15}}{30} & \frac{2}{9}+\frac{\sqrt{15}}{15} & \frac{5}{36}
\end{array}\right]
$$

and $\operatorname{det}(I-z A)=1-\frac{1}{2} z+\frac{1}{10} z^{2}-\frac{1}{120} z^{3}$.

Cooper and Vignesvaran[9] obtained the optimum values $\lambda=0.202740067$ and $\beta=1.159572736$ when solving the problem(10). For these values of $\lambda$ and $\beta, \rho[M(z)]<0.1599$ for all $z \in \mathbb{C}^{-}$.

Next it remains to choose the elements of $B=\left[b_{i j}\right]$ so that the iteration matrix $M(z)=\left[m_{i j}(z)\right]$ is strictly upper triangular matrix except that $m_{s s}(z)=$ $\phi$, a non-zero eigenvalue. For the three-stage Gauss method, the condition on $M(z)$ gives

$$
\begin{aligned}
b_{11} & =1, \\
b_{12} a_{21}+b_{13} a_{31} & =\lambda-a_{11}, \\
b_{12}\left(a_{22}-\lambda\right)+b_{13} a_{32} & =-a_{12}, \\
b_{21} b_{12}-b_{22} & =-1, \\
b_{21}\left(a_{12}-b_{12} a_{11}\right)+b_{22}\left(a_{22}-a_{21} b_{12}\right)+b_{23}\left(a_{32}-a_{31} b_{12}\right) & =\lambda, \\
b_{31} b_{12} & =0, \\
b_{31} a_{11}+b_{32} a_{21}+b_{33} a_{31} & =0 .
\end{aligned}
$$


From (13), it happens that $b_{31}=0$. Again the equations (13) together with $\operatorname{det} B=\beta$ may be solved by choosing $b_{21}=0$ and this gives

$$
B=\left[\begin{array}{ccc}
1 & 0.151290053 & 0.068750541 \\
0 & 1 & 0.058981649 \\
0 & -0.983175783 & 1.101583408
\end{array}\right] \text {. }
$$

Consider the four-stage Gauss method with matrix of coefficients $A=\left[a_{i j}\right]$ obtained by solving the sets of equations

$$
\sum_{j=1}^{4} a_{i j} c_{j}^{r-1}=\frac{c_{i}^{r}}{r}, \quad r=1,2,3,4
$$

for each $i=1,2,3,4$, where $c_{1}, c_{2}, c_{3}, c_{4}$ are the zeros of $P_{4}(2 x-1)$, the transformed legendre polynomial of degree 4 . For this method,

$$
\operatorname{det}(I-z A)=1-\frac{1}{2} z+\frac{3}{28} z^{2}-\frac{1}{84} z^{3}+\frac{1}{1680} z^{4} .
$$

The condition on $M(z)$ with the choices $b_{31}=0$ and $b_{41}=b_{42}=0$ give a system of equations which may be ordered as a sequence of sets of lnear equations given below:

$$
\begin{aligned}
& b_{11}=1, \\
& b_{12} a_{21}+b_{13} a_{31}+b_{14} a_{41}=\left(\lambda-a_{11}\right), \\
& b_{12}\left(a_{22}-\lambda\right)+b_{13} a_{32}+b_{14} a_{42}=-a_{12} \text {, } \\
& b_{12} a_{23}+b_{13}\left(a_{33}-\lambda\right)+b_{14} a_{43}=-a_{13} \text {, } \\
& +\left(b_{12} a_{31}-a_{32}\right) b_{23}+\left(b_{12} a_{41}-a_{42}\right) b_{24}=-\lambda \text {, } \\
& \left(a_{13}-b_{13} a_{11}\right) b_{21}+\left(a_{23}-b_{13} a_{21}\right) b_{22} \\
& +\left(a_{33}-b_{13} a_{31}\right) b_{23}+\left(a_{43}-b_{13} a_{41}\right) b_{24}=0 \text {, }
\end{aligned}
$$




$$
\begin{aligned}
b_{33} & =1, \\
b_{32} a_{21}+b_{34} a_{41} & =-a_{31}, \\
b_{32} a_{23}+b_{34} a_{43} & =\lambda-a_{33}, \\
b_{43} a_{31}+b_{44} a_{41} & =0 .
\end{aligned}
$$

Cooper and Vignesvaran[9] showed that these equations can be solved only for one positive value of $\lambda, \lambda=0.146840443$ and they obtained the optimum value $\beta=1.034$ to solve the problem (10). In this case, $\rho[M(z)]<0.3467$ for $\operatorname{Re}(z) \leq 0$. With these values of $\lambda$ and $\beta$, the set of equations (15),(16),(17),(18) and the equation $\operatorname{det} B=\beta$ give

$$
B=\left[\begin{array}{llll}
1 & 0.265166833 & 0.079402432 & -0.018488567 \\
0.124164683 & 1.032924356 & 0.009858978 & 0.124164683 \\
0 & -0.786754443 & 1 & -0.108118541 \\
0 & 0 & -1.109340683 & 1.045019753
\end{array}\right] .
$$

\section{Schemes with Improving Rates of Convergence}

In this section, additional constraints, which require super-linear convergence at the origin and infinity, are imposed on the spectral radius of the iteration matrix $M(z)$ in addition to the condition that $M(z)$ has only one non-zero eigenvalue. The results were obtained for the two-stage Gauss method in [22]. In this paper, new schemes corresponding to the iteration scheme (7) for threestage and four-stage Gauss methods are obtained respectively.

\subsection{The Case $\rho[M(z)]=0$ at $z=0$}

For the three-stage Gauss method, the additional constraint $\rho[M(z)]=0$ at $z=0$ gives $\beta=1$. Therefore, the other parameter $\lambda$ has to be chosen to solve 
the problem(10). It follows from (11) that the polynomial $p$ is given by

$$
p(\omega)=a_{0} \omega(1-\omega)^{2}+(1-\omega)\left[a_{1} \omega-a_{2}(1-\omega)\right]^{2},
$$

where $a_{0}=3-\frac{1}{10 \lambda^{2}}, \quad a_{1}=3-\frac{1}{2 \lambda}, \quad, \quad a_{2}=1-\frac{1}{120 \lambda^{3}}$.

A simple grid search procedure shows that good approximation to the optimum value of $\lambda$ to minimize the maximum of $p$ on $[0,1]$ is given by $\lambda=$ 0.191729022. Again the condition on $M(z)$ gives the set of equations (13) and these equations togethger with $\operatorname{det} B=\beta$ may be solved by choosing $b_{21}=0$. This gives

$$
B=\left[\begin{array}{ccc}
1 & 0.115697224 & 0.067542178 \\
0 & 1 & 0.009448755 \\
0 & -0.885047715 & 0.991637400
\end{array}\right] .
$$

In this case $\rho[M(z)]<0.2326$ for all $z \in \mathbb{C}^{-}$.

For the four-stage Gauss method, the additional constraint $\rho[M(z)]=0$ at $z=0$ gives $\beta=1$. Again from (11), the polynomial $p$ is given by

$$
p(\omega)=(1-\omega)^{2}\left[a_{4}(1-\omega)-a_{2} \omega\right]^{2}+\omega(1-\omega)\left[a_{1} \omega-a_{3}(1-\omega)\right]^{2},
$$

where $a_{1}=4-\frac{1}{2 \lambda}, a_{2}=6-\frac{3}{28 \lambda^{2}}, a_{3}=4-\frac{1}{84 \lambda^{3}}, a_{4}=1-\frac{1}{1680 \lambda^{4}}$. Again the system of equations (15),(16),(17) and (18) can be solved only for $\lambda=$ 0.146840443 and for these fixed values of $\lambda$ and $\beta$, the equations (15), (16), (17), (18)and $\operatorname{det} B=\beta$ gives

$$
B=\left[\begin{array}{lccc}
1 & 0.265166833 & 0.079402432 & -0.018488567 \\
0.124164683 & 1.032924356 & 0.009858978 & 0.124164683 \\
0 & -0.786754443 & 1 & -0.108118541 \\
0 & 0 & -1.072863330 & 1.010657402
\end{array}\right] .
$$

In this case $\rho[M(z)]<0.3542$ for all $z \in \mathbb{C}^{-}$.

The equation $|\phi(z)|=c$ describes a closed curve in the $z$-plane. Typical curves are plotted for different values of $c$ and sketched in Figures 1 and 2 for three-stage and four-stage Gauss methods respectively. In this case, $\rho[M(z)] \leq$ $c$ on and interior to the curve. Since $\rho[M(0)]=0$, these schemes are expected to perform well as typical stiff problems have Jacobian with some eigenvalues of small modulus. 

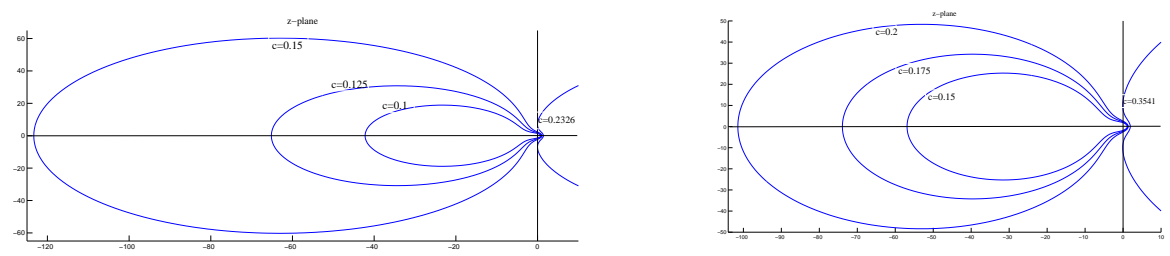

Figure 1: Curves $\rho[M(z)]=c \quad$ for $s$ Figare 2: Curves $\rho[M(z)]=c \quad$ for $s=4$

\subsection{The Case $\rho[M(z)]=0$ at $z=\infty$}

The constraint $\rho[M(\infty)]=0$ for the three-stage Gauss method gives $\lambda=\sqrt[3]{\frac{\beta}{120}}$ and the polynomial $p$, given by (11), is

$$
p(\omega)=\omega\left[a_{0} \omega-a_{2}(1-\omega)\right]^{2}+a_{1}^{2} \omega^{2}(1-\omega),
$$

where $a_{0}=1-\beta, \quad a_{1}=3-\frac{\beta}{2 \lambda}, \quad a_{2}=3-\frac{\beta}{10 \lambda^{2}}$. By search procedure, a good approximation to the optimum value of $\beta$ is obtained by $\beta=1.181387098$ and the corresponding $\lambda$ is given by $\lambda=0.214323763$. In this case $\rho[M(z)]<0.2359$ for all $z \in \mathbb{C}^{-}$. With these values of $\lambda$ and $\beta$, the equations (13) with $\operatorname{det} B=\beta$ may be solved by choosing $b_{21}=0$. This gives

$$
B=\left[\begin{array}{ccc}
1 & 0.187138824 & 0.071808998 \\
0 & 1 & 0.112237507 \\
0 & -0.958395854 & 1.073819136
\end{array}\right]
$$

For the four-stage Gauss method, the additional constraint $\rho[M(\infty)]=0$ gives $\beta=1680 \lambda^{4}$. It follows from (11) that the polynomial $p$ is given by

$$
p(\omega)=\left[a_{0} \omega^{2}-a_{2} \omega(1-\omega)\right]^{2}+\omega(1-\omega)\left[a_{1} \omega-a_{3}(1-\omega)\right]^{2},
$$

where $a_{0}=1-\beta, \quad a_{1}=4-\frac{\beta}{2 \lambda}, \quad a_{2}=6-\frac{3 \beta}{28 \lambda^{2}}, \quad a_{3}=4-\frac{\beta}{84 \lambda^{3}}$. With the value $\lambda=0.146840443$, which solves the sets of equations 15),(16),(17),(18), and the corresponding value of $\beta$, those sets of equations and $\operatorname{det} B=\beta$ give 


$$
B=\left[\begin{array}{lccc}
1 & 0.265166833 & 0.079402432 & -0.018488567 \\
0.124164683 & 1.032924356 & 0.009858978 & 0.124164683 \\
0 & -0.786754443 & 1 & -0.108118541 \\
0 & 0 & -0.837985352 & 0.789397936
\end{array}\right]
$$

In this case $\rho[M(z)]<0.2189$ for all $z \in \mathbb{C}^{-}$.
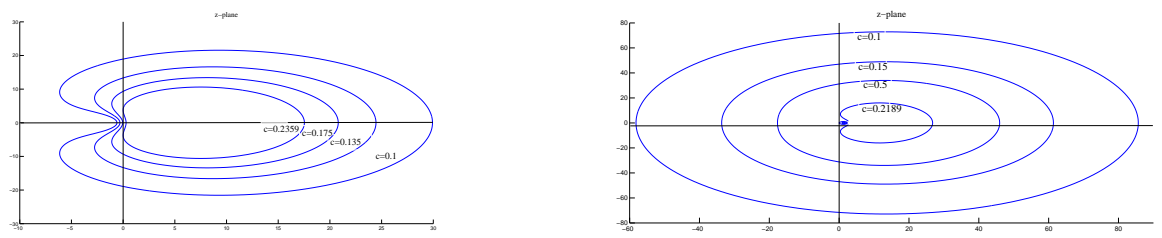

Figure 3: Curves $\rho[M(z)]=c \quad$ for $s$ FigBre 4: Curves $\rho[M(z)]=c \quad$ for $s=4$

As per the plotted curves for $\rho[M(z)]=c$ for different values of $c$ in in Figures 3 and 4 for three-stage and four-stage Gauss methods, these schemes are expected to perform well as typical stiff problems have Jacobian with some eigenvalues of large negative real parts and $\rho[M(\infty)]=0$.

\section{Numerical Results}

To evaluate the efficiency of the schemes obtained here, a range of numerical experiments was carried out. For each experiment, a single step was carried out, in each case, using the Jacobian evaluated at the initial point. For each scheme tested, the initial iterate $Y^{0}$ is chosen as $Y^{0}=e \otimes x$, where $x$ is the true solution at the initial point.

Problem 1 denotes the non-linear system given by [14]

$$
\begin{array}{ll}
x_{1}^{\prime}=-0.013 x_{1}+1000 x_{1} x_{3}, & x_{1}(0)=1 \\
x_{2}^{\prime}=2500 x_{2} x_{3}, & x_{2}(0)=1 \\
x_{3}^{\prime}=0.013 x_{1}-1000 x_{1} x_{3}-2500 x_{2} x_{3}, & x_{3}(0)=0
\end{array}
$$

where the eigenvalues of the Jacobian at the initial point are $0,-0.0093$ and -3500 . 
Problem 2 is the elliptic two-body problem, with eccentricity 0.6 ,

$$
\begin{array}{ll}
x_{1}^{\prime}=x_{3}, & x_{1}(0)=0.4, \\
x_{2}^{\prime}=x_{4}, & x_{2}(0)=0, \\
x_{3}^{\prime}=-x_{1}\left(x_{1}^{2}+x_{2}^{2}\right)^{-3 / 2}, & x_{3}(0)=0, \\
x_{4}^{\prime}=-x_{2}\left(x_{1}^{2}+x_{2}^{2}\right)^{-3 / 2}, & x_{4}(0)=2 .
\end{array}
$$

The eigenvalues at the initial point are \pm 5.5902 and $\pm 3.9528 i$.

Problem 3 is the HIRES problem given by [18],

$$
\begin{array}{ll}
x_{1}^{\prime}=-1.71 x_{1}+0.43 x_{2}+8.32 x_{3}+0.0007, & x_{1}(0)=1, \\
x_{2}^{\prime}=1.71 x_{1}-8.75 x_{2}, & x_{2}(0)=0, \\
x_{3}^{\prime}=-10.03 x_{3}+0.43 x_{4}+0.035 x_{5}, & x_{3}(0)=0, \\
x_{4}^{\prime}=8.32 x_{2}+1.71 x_{3}-1.12 x_{4}, & x_{4}(0)=0, \\
x_{5}^{\prime}=-1.745 x_{5}+0.43 x_{6}+0.43 x_{7}, & x_{5}(0)=0, \\
\left.x_{6}^{\prime}=-280 x_{6} x_{8}+0.69 x_{4}+1.71 x_{5}\right)-0.43 x_{6}+0.69 x_{7}, & x_{6}(0)=0, \\
x_{7}^{\prime}=280 x_{6} x_{8}-1.81 x_{7}, & x_{7}(0)=0, \\
x_{8}^{\prime}=-x_{7}^{\prime}, & x_{8}(0)=0.0057 .
\end{array}
$$

The eigenvalues of the Jacobian at the initial point are $0,-10.4841$, $-8.278,-0.2595,-0.5058,-2.3147$ and $-2.6745 \pm 0.1499 i$.

Problem 4 denotes the system

$$
\begin{array}{ll}
x_{1}^{\prime}=x_{2}, & x_{1}(0)=2, \\
x_{2}^{\prime}=10^{6}\left(\left(1-x_{1}^{2}\right) x_{2}\right)-x_{1}, & x_{2}(0)=0,
\end{array}
$$

derived from the Van der Pol's equation and given by [11]. The eigenvalues of the Jacobian at the initial point are close to 0 and -3000000 .

Problem 5 denotes the system, with non-linear coupling between smooth and transient components,

$$
\begin{array}{ll}
x_{1}^{\prime}=-10^{5} x_{1}+2, & x_{1}(0)=1, \\
x_{2}^{\prime}=-10^{6} x_{2}+0.1 x_{1}^{2}, & x_{2}(0)=1, \\
x_{3}^{\prime}=-40 \times 10^{5} x_{3}+0.4\left(x_{1}^{2}+x_{2}^{2}\right), & x_{3}(0)=1, \\
x_{4}^{\prime}=-10^{7} x_{4}+x_{1}^{2}+x_{2}^{2}+x_{3}^{2}, & x_{4}(0)=1,
\end{array}
$$

where the Jacobian has constant eigenvalues $-10^{5},-10^{6},-40 \times 10^{5}$ and $-10^{7}$.

For each problem, a single step was carried out, in each case, using the Jacobian evaluated at the initial point . For each scheme tested, the initial iterate $Y^{0}$ is chosen as $Y^{0}=e \otimes x$, where $x$ is the true solution at the initial point. 


\begin{tabular}{|c|c|c|c|c|}
\hline$e_{m}$ & Method 1 & Method 1* & Method 2 & Method 2* \\
\hline$e_{1}$ & 0.000956220 & 0.000824833 & 0.000895782 & 0.000866327 \\
$e_{2}$ & 0.000152341 & 0.000110398 & 0.000142783 & 0.000143328 \\
$e_{3}$ & 0.000024273 & 0.000000910 & 0.000028768 & 0.000028367 \\
$e_{4}$ & 0.000003867 & 0.000000031 & 0.000001011 & 0.000000127 \\
$e_{5}$ & 0.000000616 & 0.000000005 & 0.000000054 & 0.000000033 \\
$e_{6}$ & 0.000000098 & 0.000000001 & 0.000000016 & 0.000000008 \\
$e_{7}$ & 0.000000016 & 0.000000000 & 0.000000005 & 0.000000002 \\
$e_{8}$ & 0.000000002 & & 0.000000001 & 0.000000001 \\
$e_{9}$ & 0.000000000 & & 0.000000000 & \\
\hline
\end{tabular}

Table 1: Values of $e_{m}$ for Problem 1 with $h=0.1$

Method 1 denotes the three-stage Gauss method implemented according to the iteration scheme(7) with $\lambda=0.202740067$ and the matrix $B$ given by (14). Method $1^{*}$ is the same method implemented using the scheme (7) with $\lambda=0.191729022$ and $B$ given by (20) for the case $\rho[M(z)]=0$ at $z=$ 0 . Method $1^{* *}$ is also the same method implemented using the scheme (7) with $\lambda=0.214323763, B$ given by $(22)$ for the case $\rho[M(z)]=0$ at $z=\infty$. Method 2 denotes the four-stage Gauss method implemented according to the scheme (7) with $\lambda=0.146840443$ and $B$ given by (19). Method $2^{*}$ is the same method implemented using the scheme (7) with $\lambda=0.146840443$ and $B$ given by $(21)$ for $\rho[M(0)]=0$. Method $2^{* *}$ is also the same method implemented using the scheme (7) with the same value of $\lambda$ and $B$ given by (23) for $\rho[M(\infty)]=0$.

For each method and problem, the quantities

$$
e_{m}=\left\|E^{m}\right\|, \quad m=1,2,3, \cdots
$$

were computed using the maximum norm on $\mathbb{R}^{n s}$. The values $e_{m}$ for which $e_{m} \leq \mathrm{TOL}=10^{-9}$ are tabulated for each problem and method. Similar results are obtained for different values of TOL. The results are given below for each problem for three-stage and four-stage Gauss methods. 


\begin{tabular}{|c|c|c|c|c|}
\hline$e_{m}$ & Method 1 & Method $1^{*}$ & Method 2 & Method 2* \\
\hline$e_{1}$ & 0.064323263 & 0.055470109 & 0.060234720 & 0.058254081 \\
$e_{2}$ & 0.010337141 & 0.007429666 & 0.009595467 & 0.009632142 \\
$e_{3}$ & 0.001670882 & 0.000067048 & 0.001945151 & 0.001918104 \\
$e_{4}$ & 0.000270379 & 0.000000270 & 0.000072013 & 0.000008450 \\
$e_{5}$ & 0.000043831 & 0.000000002 & 0.000002754 & 0.000000149 \\
$e_{6}$ & 0.000007117 & 0.000000000 & 0.000000106 & 0.000000000 \\
$e_{7}$ & 0.000001157 & & 0.000000004 & \\
$e_{8}$ & 0.000000189 & & 0.000000000 & \\
$e_{9}$ & 0.000000031 & & & \\
$e_{10}$ & 0.000000005 & & & \\
$e_{11}$ & 0.000000001 & & & \\
\hline
\end{tabular}

Table 2: Values of $e_{m}$ for Problem 2 with $h=0.01$

\begin{tabular}{|c|c|c|c|c|}
\hline$e_{m}$ & Method 1 & Method $1^{*}$ & Method 2 & Method 2* \\
\hline$e_{1}$ & 0.017382122 & 0.015000547 & 0.016278083 & 0.015742827 \\
$e_{2}$ & 0.002728084 & 0.002012693 & 0.002608108 & 0.002618024 \\
$e_{3}$ & 0.000428244 & 0.000013213 & 0.000523517 & 0.000516215 \\
$e_{4}$ & 0.000067235 & 0.000000021 & 0.000017567 & 0.000003710 \\
$e_{5}$ & 0.000010557 & 0.000000000 & 0.000000591 & 0.000000025 \\
$e_{6}$ & 0.000001658 & & 0.000000020 & 0.000000000 \\
$e_{7}$ & 0.000000260 & & 0.000000001 & \\
$e_{8}$ & 0.000000041 & & & \\
$e_{9}$ & 0.000000006 & & & \\
$e_{10}$ & 0.000000001 & & & \\
$e_{11}$ & 0.000000000 & & & \\
\hline
\end{tabular}

Table 3: Values of $e_{m}$ for Problem 3 with $h=0.01$

\section{Concluding Remarks}

According to the numerical results, for three-stage Gauss method, the method $1^{*}$ performs better than method 1 for the problems whose Jacobian matrices have small eigenvalues and the method $1^{* *}$ performs better than method 1 for the problems whose Jacobian matrices have eigenvalues with large negative real part. For four-stage Gauss method, Method $2^{*}$ is better than Method 2 for 


\begin{tabular}{|c|c|c|c|c|}
\hline$e_{m}$ & Method 1 & Method 1** & Method 2 & Method 2** \\
\hline$e_{1}$ & 0.000000820 & 0.000000840 & 0.000000884 & 0.000000876 \\
$e_{2}$ & 0.000000149 & 0.000000155 & 0.000000364 & 0.000000275 \\
$e_{3}$ & 0.000000024 & 0.000000018 & 0.000000119 & 0.000000007 \\
$e_{4}$ & 0.000000004 & 0.000000000 & 0.000000039 & 0.000000001 \\
$e_{5}$ & 0.000000001 & & 0.000000013 & 0.000000000 \\
$e_{6}$ & & & 0.000000004 & \\
$e_{7}$ & & & 0.000000001 & \\
$e_{8}$ & & & 0.000000001 & \\
\hline
\end{tabular}

Table 4: Values of $e_{m}$ for Problem 4 with $h=0.1$

\begin{tabular}{|c|c|c|c|c|}
\hline$e_{m}$ & Method 1 & Method 1 & Method 2 & Method 2** \\
\hline$e_{1}$ & 1.229888995 & 1.259710539 & 1.325937141 & 1.313889816 \\
$e_{2}$ & 0.223847832 & 0.232791462 & 0.546093036 & 0.412513120 \\
$e_{3}$ & 0.035719849 & 0.026955933 & 0.177844840 & 0.010989760 \\
$e_{4}$ & 0.005699876 & 0.000005372 & 0.057918610 & 0.000015235 \\
$e_{5}$ & 0.000909531 & 0.000000009 & 0.018862359 & 0.000000018 \\
$e_{6}$ & 0.000145134 & 0.000000001 & 0.006142907 & 0.000000000 \\
$e_{7}$ & 0.000023159 & 0.000000000 & 0.002000561 & \\
$e_{8}$ & 0.000003696 & & 0.000651523 & \\
$e_{9}$ & 0.000000590 & & 0.000212182 & \\
$e_{10}$ & 0.000000094 & & 0.000069101 & \\
$e_{11}$ & 0.000000015 & & 0.000022504 & \\
$e_{12}$ & 0.000000002 & & 0.000007329 & \\
$e_{13}$ & 0.000000000 & & 0.000002387 & \\
$e_{14}$ & & & 0.000000777 & \\
$e_{15}$ & & & 0.000000253 & \\
\hline
\end{tabular}

Table 5: Values of $e_{m}$ for Problem 5 with $h=0.1$

problems with small eigenvalues and Method $2^{* *}$ is better than Method 2 for problems with eigenvalues which have large negative real parts. In overall, the numerical experiments confirm that the new schemes obtained for the Gauss methods peform well. 


\section{References}

[1] T.A.Bickart, An efficient solution process for implicit RungeKutta methods, SIAM J. Numer.Anal., 14 (1977), 1022-1027. doi: http://dx.doi.org/10.1137/0714069

[2] J.C.Butcher, On the implementation of implicit Runge-Kutta methods, BIT, 16 (1976), 237-240. doi: 10.1007/BF01932265

[3] J.C.Butcher, Some implementation schemes for implicit Runge-Kutta methods, Proceeding of the Dundee Conference on Numerical Analysis ,Lecture Notes in Mathematics, Springer-Verlag, 773,(1980), 12-24. doi: 10.1007/BFb0094160

[4] J.C.Butcher, J.R.Cash, Towards efficient Runge-Kutta methods for stiff systems, SIAM J.Numer. Anal., 27 (1990), 753-761. doi: http://dx.doi.org/10.1137/0727044

[5] J.R.Cash, On a class of implicit Runge-Kutta procedures, IMA J. Appl. Math., 19 (1977), 455-470. doi: 10.1093/imamat/19.4.455

[6] F.H.Chipman, The implementation of Runge-Kutta implicit processes, BIT, 13 (1973), 391-393. doi: 10.1007/BF01933400

[7] A.G.Collings and G.J.Tee, An analysis of Euler and implicit Runge-Kutta numerical integration schemes for structural dynamic problems, Proceeding of the Sixth Australasian Conference on the Mechanics of Structures and Materials, 1 (1977), 147-154.

[8] G.J.Cooper and J.C.Butcher, An iteration scheme for implicit RungeKutta methods, IMA J. Numer. Anal., 3 (1983), 127-140. doi: 10.1093/imanum/3.2.127

[9] G.J.Cooper and R. Vignesvaran, A scheme for the implementation of implicit Runge-Kutta methods, Computing, 45 (1990), 321-332. doi: 10.1007/BF02238800

[10] G.J.Cooper and R. Vignesvaran, Some schemes for the implementation of implicit Runge-Kutta methods, J. Comp. App. Math., 45 (1993), 213-225. doi: http://dx.doi.org/10.1016/0377-0427(93)90276-H

[11] H.T.Davis, Introduction to non-linear differential and integral equations, Dover, New York (1962). 
[12] W.H. Enright, Improving the efficiency of matrix operations in the numerical solution of ODEs, Technical Report 98 , Computer Science Dept., Univ. of Toronto (1976).

[13] R. Frank and C.W. Ueberhuber, Iterated defect correction for the efficient solution of stiff systems of ordinary differential equations, BIT, 17 (1977), 146-159. doi: 10.1007/BF01932286

[14] C.W. Gear, The automatic integration of stiff ordinary differential equations, Proc. IFIP Congress, (1968), 187-193.

[15] I. Gladwell and R.M. Thomas, Efficiency of methods for second order problems, IMA J. Numer. Anal., 10 (1990), 181-207. doi: 10.1093/imanum/10.2.181

[16] S. Gonzalez, C. Gonzalez and J.I. Montijano, Iterative schemes for Gauss methods, Comp. Math. Applic., 27 (1994), 67-81. doi: http://dx.doi.org/10.1016/0898-1221(94)90150-3

[17] S. Gonzalez, J.I. Montijano and L. Randez, Iterative schemes for three stage implicit Runge-Kutta methods, App. Numer. Math., 17 (1995), 363382. doi: http://dx.doi.org/10.1016/0168-9274(95)00070-B

[18] E.Hairer and G.Wanner, Solving ordinary differential equations II: Stiff and Differential algebraic problems, Springer-Verlag, Berlin (1996).

[19] K.D. Peat and R.M. Thomas, Implementation of Iteration schemes for Implicit Runge-Kutta Methods, Numerical Analysis Report No. 169 , Dept. of Mathematics, University of Manchester (1989).

[20] R.Vigneswaran, Improving Rates of Convergence of Iterative Schemes for Implicit Runge-Kutta Methods, Applied Numerical Analysis and Computational Mathematics., 1 (2004), 327-338. doi: 10.1002/anac.200310029

[21] R.Vigneswaran, Some Linear Schemes for Two-stage Gauss Type RungeKutta Method, Proceeding of the Third Annual Research Session at the Eastern University, Sri Lanka, (2004), 160-183.

[22] R. Vigneswaran, Some Efficient Schemes with Improving Rate of Convergence for Two stage Gauss Method, Journal of Mathematics, Statistics and Operational Research(JMSOR), 2 (2013), 23-29. 